\title{
Budget global de la santé: un problème déguisé en solution
}

\section{Udo Schulte}

Dr méd., spécialiste en médecine générale

\author{
Dans les pays avec des systèmes de sécurité sociale, les tentatives d'influencer la \\ répartition des primes d'assurance encaissées auprès des assurés font partie du jeu \\ politique. Par contre, le bénéfice que les assurés et les patients peuvent en retirer \\ est souvent discutable. Le budget global appliqué depuis 30 ans en Allemagne le \\ montre bien.
}

Dans les systèmes de soins modernes, les coûts engendrés par les maladies graves dépassent les capacités individuelles. Ils sont donc garantis par les patients potentiels, ce qui engendre des flux monétaires importants: or, le problème est plus complexe qu'il n'y paraît. Même des démocraties très développées comme les Etats-Unis ne parviennent actuellement pas à le résoudre.

Les acteurs politiques tendent à présenter des solutions simples aux problèmes complexes. En ce sens, l'Allemagne a introduit en 1989 un budget global limitant actuellement les dépenses pour les traitements ambulatoires et hospitaliers à $15,2 \%$ de la somme salariale. Alors qu'aucun effet positif n'a été démontré sur l'évolution globale des coûts, cette enveloppe budgétaire entraîne de toute évidence de nombreuses distorsions. Malgré des réformes législatives du système tous les deux ans, le budget global a un impact défavorable sur la structure des soins. Ces réformes sont présentées ici à partir du groupe des ophtalmologues (autrefois petit et homogène) et du groupe des généralistes (autrefois grand et hétérogène).

\section{Pénurie d'ophtalmologues sans activité chirurgicale pour cause d'enveloppe budgétaire}

En 1998, un ophtalmologue installé dans le Land du Bade-Wurtemberg, exerçant sans activité chirurgicale, touchait dans le cadre du budget global $€ 33,95$ par trimestre pour le suivi d'un patient. En 2015, il percevait $€$ 33,54 pour le même travail. Sur la base d'un renchérissement de 2,5\% par année, cela équivaut à une perte de revenu réelle de $36 \%$.

La cause de cette évolution réside dans la budgétisation des prestations ophtalmologiques et l'émergence d'opé- rations ambulatoires, dont la rémunération nettement plus élevée provient du même budget bloqué. C'est pourquoi l'ophtalmologue sans activité chirurgicale enregistre aujourd'hui des recettes trimestrielles moyennes d'environ $€ 48600$, contre $€ 131000$ pour celui qui pratique des interventions chirurgicales.

La pénurie de relève (induite) pour les cabinets ophtalmologiques sans activité chirurgicale provoque aujourd'hui des temps d'attente dépassant six mois. Les patients sont très mécontents de cette situation, mais ne sont pas en mesure d'en attribuer la responsabilité aux vrais responsables, c'est-à-dire aux décideurs politiques, tant le système de santé allemand est opaque et complexe. Parallèlement, les délais d'attente pour les implants de lentilles ne sont que de quelques semaines.

\section{Pénurie de généralistes pour cause d'enveloppe budgétaire}

Chez les généralistes du Land du Bade-Wurtemberg, en 2015 , l'indemnisation par patient pour la prise en charge globale sur trois mois se situait $32 \%$ en dessous du niveau de 1996 (sur la base des mêmes estimations de renchérissement). Au début, cette baisse était liée au financement de prestations techniques coûteuses de spécialistes sur le budget des généralistes. Entre-temps, on y a mis fin, mais la systématique budgétaire très complexe continue de creuser l'écart entre les revenus des médecins de premier recours et ceux des spécialistes. Au lieu d'une compensation de ces pertes de revenus liées à un budget global, les généralistes ont eu droit, dans le meilleur des cas, à un peu d'estime sociale, dans le pire des cas, au discours habituel de légitimation politique, voire même à des envolées lyriques sur l'importance du médecin de campagne. 
La réalité en médecine de premier recours se rapproche de celle des ophtalmologues sans activité chirurgicale: sur cinq généralistes âgés de 65 ans, les chiffres montrent qu'un seul sera remplacé par un médecin fraîchement diplômé. Dans les faits, au vu de la situation économique et démographique, il s'agit plutôt d'un médecin sur huit à dix qui est remplacé par un plus jeune, car seul un petit nombre d'entre eux s'engage dans le domaine peu valorisé de la prise en charge des patients. De plus, la part croissante des femmes dans la profession s'accompagne d'une offre de temps de travail réduite. Dix pour cent des généralistes installés continuent de travailler au-delà de l'âge de la retraite par manque de relève. Sans renversement de cette tendance, dans dix ans, le nombre total de généralistes sera, même dans le scénario le plus optimiste, nettement inférieur à 50\% de l'effectif actuel. Avec une telle baisse des effectifs, la structure des soins de premier recours perdra sa capacité de fonctionnement et disparaîtra tôt ou tard.

\section{Autres dommages liés au budget global}

Les dommages consécutifs au budget global allemand présentés ici s'appliquent à toutes les disciplines dont la création de valeur se fonde principalement sur la force de travail du médecin. En raison des économies d'échelle, ils ne s'appliquent pas aux disciplines qui peuvent déléguer une part importante de leur travail, comme par exemple la médecine de laboratoire. Quant aux disciplines très techniques ou pharmaceutiques, elles réclament le refinancement de leurs investissements, une revendication acceptée par le politique, et comptent donc, dans le sillage de la budgétisation, parmi les profiteurs du système.

En Allemagne, si le pilotage politique n'a pas permis d'établir, même à long terme, une égalité de traitement entre les disciplines, il a même cimenté une inégalité entre les régions: les indemnisations de toutes les disciplines dans les 17 régions d'approvisionnement de la République fédérale varient fortement: un médecin généraliste dans le Land de Thuringe touche $€ 64,34$ par trimestre et patient pour la prise en charge globale, un généraliste de Hambourg reçoit un tiers de moins pour la même prestation. Ici aussi, il s'agit d'une conséquence de l'influence politique sur la répartition d'un budget qui n'est clairement défini qu'en théorie.

Les promesses d'économie sont suivies d'allocations erronées et d'excès bureaucratiques.

Normalement, la mise en place d'un budget est politiquement très facile à réaliser. Les acteurs agissent souvent avec l'aide d'une machine politico-médiatique qui leur est dévouée, car économiser de l'argent pour les citoyens est une promesse électorale porteuse. Aux yeux de l'élec- teur, la fixation d'une limite des dépenses anonyme, apparemment sans conséquences, que d'autres doivent respecter, est une offre qu'il ne peut refuser - parce qu'il ne voit généralement pas que c'est son propre approvisionnement en soins qui sera sensiblement réduit. Le passage au budget global en Allemagne reste un problème non résolu, même après 30 ans de bricolages incessants - aussi et surtout à cause de l'influence d'intérêts économiques et de redistribution, étrangers à la médecine. Ceux-ci interfèrent avec l'évolution démographique, médicale et industrielle et produisent toujours plus de nouvelles distorsions et allocations erronées qui corrompent le système et engendrent des contraintes réglementaires inimaginables pour les personnes extérieures au système. Les coûts de cette bureaucratie ne sont présentés nulle part de façon transparente.

\section{L'agenda caché: l'emprise politique sur les primes des assurés pour imposer des intérêts étrangers à la médecine}

Cette enveloppe budgétaire ne vise donc pas à résoudre le problème, mais permet l'accès politique et opaque à plus de 200 milliards d'euros de cotisations obligatoires de l'assurance-maladie - un montant qui correspond à $2 / 3$ du budget fédéral financé par les contribuables. Contrairement aux impôts, les cotisations des caissesmaladie sont considérées comme plus justes et encaissées de façon soi-disant transparente pour «une bonne cause».

S'arroger le pouvoir de distribuer de telles sommes ouvre des possibilités d'influence inattendues pour les représentants d'intérêts de tous bords. Les responsables de la politique familiale financent ainsi l'exonération des membres d'une famille sans activité professionnelle, les spécialistes de la politique sociale subventionnent les cotisations des retraités, des bénéficiaires de l'aide sociale et des requérants d'asile avec d'immenses sommes financées par les assurés réguliers; les politiciens proche de l'industrie font le bonheur de leur clientèle avec des solutions informatiques coûteuses imposées aux cabinets médicaux, mais dysfonctionnelles. Le complexe politico-pharmaceutique aussi mériterait à lui seul un chapitre.

Le principal gagnant du système budgétaire allemand est cette nébuleuse de la justice, de l'administration et des représentants d'autres acteurs qui promettent à leurs mandants, moyennant une bonne rémunération, de les conduire sans trop d'ecchymoses à travers le combat politico-administratif pour répartir les parts budgétaires. Les perdants sont les patients et les forces productives du système de santé: les médecins et leurs nombreux collaborateurs. 